\title{
Désinfection à la chaleur humide des respirateurs N95, inactivation du SRAS-CoV-2 et effets sur les propriétés des respirateurs
}

\author{
Simeon C. Daeschler MD, Niclas Manson MSP, Kariym Joachim MSc, Alex W.H. Chin PhD, Katelyn Chan MScS, \\ Paul Z. Chen BScA, Kiana Tajdaran MD, Kaveh Mirmoeini MD, Jennifer J. Zhang MD PhD, Jason T. Maynes MD PhD, \\ Libo Zhang MD, Michelle Science MD, Ali Darbandi PhD, Derek Stephens MSc, Frank Gu PhD, Leo L.M. Poon PhD, \\ Gregory H. Borschel MD
}

- Citation : CMAJ 2020 October 13;192:E1189-97. doi : 10.1503/cmaj.201203-f; diffusion hâtive le 30 juillet 2020

Voir la version anglaise de l'article ici : www.cmaj.ca/lookup/doi/10.1503/cmaj.201203

\section{RÉSUMÉ}

CONTEXTE : La demande sans précédent de respirateurs N95 durant la pandémie de maladie à coronavirus 2019 (COVID19) a entraîné une pénurie mondiale. Nous avons validé un protocole de décontamination rapide et économique répondant aux normes réglementaires afin de permettre la réutilisation sûre de ce type de masque.

MÉTHODES : Nous avons contaminé 4 modèles courants de respirateurs N95 avec le coronavirus du syndrome respiratoire aigu sévère 2 (SRAS-CoV-2) et avons évalué l'inactivation virale après une désinfection de 60 minutes à $70^{\circ} \mathrm{C}$ et à une humidité relative de $0 \%$. De même, nous avons étudié l'efficacité de la désinfection thermique, à une humidité relative allant de $0 \%$ à $70 \%$, de masques contaminés à Escherichia coli. Enfin, nous avons examiné des masques soumis à de multiples cycles de désinfection thermique : nous avons évalué leur intégrité structurelle à l'aide d'un microscope à balayage, et leurs propriétés protectrices au moyen des normes du National Institute for Occupational Safety and Health des États-Unis relatives à la filtration particulaire, à la résistance respiratoire et à l'ajustement.

RÉSULTATS : Une seule désinfection thermique a suffi pour que le SRASCoV-2 ne soit plus décelable sur les masques étudiés. En ce qui concerne les masques contaminés à $E$. coli, une culture de 24 heures a révélé que la bactérie n'était pratiquement plus décelable sur les masques désinfectés à $70^{\circ} \mathrm{C}$ et à une humidité relative de $50 \%$, contrairement aux masques non désinfectés (densité optique à une longueur d'onde de $600 \mathrm{~nm}: 0,02 \pm 0,02$ contre $2,77 \pm 0,09 ; p<0,001$ ), mais qu'elle persistait sur les masques traités à une humidité relative moindre. Les masques ayant subi 10 cycles de désinfection avaient toujours des fibres de diamètre semblable à celui des fibres des masques non traités, et ils répondaient encore aux normes d'ajustement, de filtration et de résistance respiratoire.

INTERPRÉTATION : La désinfection thermique a réussi à décontaminer les respirateurs N95 sans compromettre leur intégrité structurelle ni modifier leurs propriétés. Elle pourrait se faire dans les hôpitaux et les établissements de soins de longue durée avec de l'équipement facilement accessible, ce qui réduirait la pénurie de N95.
$\mathbf{L}$ a pandémie de maladie à coronavirus 2019 (COVID-19) a pris d'assaut de nombreux systèmes de santé dans le monde. En même temps, la demande sans précédent d'équipement de protection individuelle (EPI) a épuisé les réserves et brisé les chaînes d'approvisionnement mondiales en respirateurs N95. À l'heure actuelle, les travailleurs de la santé de première ligne représentent plus de $10 \%$ des personnes infectées par le coronavirus du syndrome respiratoire aigu sévère 2 (SRAS-CoV-2) dans certaines régions, et on s'attend à ce que cette proportion augmente si les réserves d'EPI continuent à diminuer ${ }^{1,2}$. Par conséquent, la protection des travailleurs de première ligne contre le SRAS-CoV-2 est aujourd'hui une préoccupation mondiale urgente ${ }^{3}$.

Les respirateurs N95 à usage unique protègent ceux qui les portent des particules infectieuses aéroportées, donc sont essentiels pour les travailleurs de première ligne pendant la pandémie de COVID-193. Cependant, la pénurie mondiale d'EPI qui sévit actuellement a forcé les organismes de réglementation à revoir leurs mesures de lutte contre les infections. Avant la pandémie, les lignes directrices recommandaient la mise au 
rebut des respirateurs $\mathrm{N} 95$ après tout contact avec un patient. Aujourd'hui, elles recommandent l'utilisation du même masque tout au long d'un quart de travail, et parfois plus longtemps ${ }^{4}$. Or, la réutilisation des masques à usage unique dans les milieux où l'exposition à des agents pathogènes aéroportés est élevée par exemple lors de la réalisation d'interventions médicales générant des aérosols auprès de patients atteints de la COVID19 - pourrait entraîner l'accumulation de matières contagieuses sur la surface des masques, mettant en péril la santé et la sécurité du personnel et des patients ${ }^{5,6}$. L'inactivation des agents pathogènes accumulés sur les respirateurs à usage unique par une technique qui maintiendrait les propriétés protectrices de ces derniers pourrait permettre une réutilisation sûre et aider à contrer temporairement la pénurie. Mais pour le moment, on croit que les méthodes de stérilisation régulièrement employées dans les établissements de santé peuvent dégrader les respirateurs à usage unique et, par le fait même, nuire à leur bon ajustement et à leur capacité de filtration?

La désinfection thermique est possiblement une solution au problème, et se veut une stratégie de décontamination des respirateurs à usage unique largement accessible et économique. Des études récentes montrent que le SRAS-CoV-2 est très sensible à la chaleur : 5 minutes à $70^{\circ} \mathrm{C}$ suffisent à l'inactiver ${ }^{5,8}$. Les microfibres de polypropylène des respirateurs N95 offerts sur le marché ont un point de dégradation supérieur à $130{ }^{\circ} \mathrm{C}$, ce qui laisse croire que le filtre de ces masques pourrait résister à une exposition répétée à une température de $70{ }^{\circ} \mathrm{C}^{9,10}$. Cependant, l'efficacité antivirale de la désinfection thermique à éliminer le SRAS-CoV-2 sur les respirateurs N95 et l'effet protecteur des respirateurs traités à la chaleur n'ont pas été validés selon les normes réglementaires élevées des États-Unis.

Nous avons cherché à savoir si la désinfection thermique à $70{ }^{\circ} \mathrm{C}$ pendant 60 minutes inactive les agents pathogènes, dont le SRAS-CoV-2, et si les propriétés protectrices essentielles des respirateurs N95 sont maintenues après de multiples cycles de désinfection et de réutilisation dans un contexte réel.

\section{Méthodes}

\section{Protocole de désinfection thermique des respirateurs N95}

Nous avons réalisé des cycles de désinfection thermique de 60 minutes à $70{ }^{\circ} \mathrm{C}$ et à une humidité relative de $0 \%$ ou de $50 \%$ sur 4 modèles de respirateurs N95 couramment offerts sur le marché (8110S, 9105S, 8210 et $1860 \mathrm{~S}$, de $3 \mathrm{M}$ ). Nous avons d'abord placé les respirateurs dans des pochettes de stérilisation (Steril-Peel, de GS Medical Packaging). Afin de contrôler la température et les conditions hygrométriques, nous avons réglé l'armoire chauffante avec humidité BevLes (BevLes inc.) à $70^{\circ} \mathrm{C}$ et à une humidité relative de $0 \%$ ou de $50 \%$. Nous avons également eu recours à un thermo-hygromètre numérique (Hagen Group inc., Canada) comme mesure supplémentaire de contrôle de la qualité. De plus, nous avons reproduit les fluctuations potentielles de température dans le monde réel en laissant refroidir les masques à température ambiante pendant 5 minutes à mi-cycle.

\section{Inactivation du SRAS-CoV-2}

Nous avons évalué l'inactivation du SRAS-CoV-2 sur les 4 modèles de N95 étudiés. Nous avons coupé des N95 non traités et des N95 ayant subi 10 traitements à la chaleur en morceaux de $1 \mathrm{~cm}^{2}$, puis avons contaminé la surface extérieure de 3 échantillons par modèle ( $n=3$ pour chaque type de respirateur) avec $5 \mu \mathrm{L}$ de SRAS-CoV-2 (environ 7,8 log dose infectieuse en culture tissulaire $50 \%$ par $\mathrm{mL}\left[\mathrm{DICT}_{50} / \mathrm{mL}\right]$ ) dans un laboratoire de sécurité biologique de niveau 3. Nous avons soumis les respirateurs contaminés à une désinfection thermique de 60 minutes à $70^{\circ} \mathrm{C}$ et à une humidité relative de $0 \%$, avec ou sans refroidissement pendant 5 minutes à mi-cycle, puis les avons placés dans un milieu de transport viral de $300 \mu \mathrm{L}$ pendant 30 minutes pour effectuer une élution virale. Nous avons ensuite titré les particules virales infectieuses recueillies, au moyen d'une évaluation normalisée de la $\mathrm{DICT}_{50}$ sur des cellules Vero E6, comme décrit par Chin ${ }^{5}$. Des échantillons de respirateurs contaminés non traités à la chaleur ont servi de témoins.

\section{Inactivation bactérienne}

Dans le but de tester l'inactivation bactérienne, nous avons coupé des $\mathrm{N} 95$ non traités (1860S, de 3M) en morceaux de $1 \mathrm{~cm}^{2}$, puis en avons contaminé la surface extérieure avec $100 \mu \mathrm{l}$ d'une solution à base d'Escherichia coli $(4 \times 108$ unités formant des colonies [UFC] par $\mathrm{mL}$; densité optique de 0,612 à une longueur d'onde de $600 \mathrm{~nm}$ ). Nous avons aussi «contaminé » un témoin négatif avec un milieu Luria-Bertani pur. Les échantillons contaminés ont été traités pendant 60 minutes à une température de $70{ }^{\circ} \mathrm{C}$ et à une humidité relative de $0 \%$, de $25 \%$, de $40 \%$ ou de $50 \%$ ( $n=4$ pour chaque condition). Nous avons également traité un échantillon à $90{ }^{\circ} \mathrm{C}$ et à une humidité relative de $70 \%$, et avons laissé un échantillon témoin contaminé à $E$. coli à température ambiante pendant 1 heure. Nous avons ensuite lavé individuellement les échantillons de $\mathrm{N} 95$ dans $1 \mathrm{~mL}$ de milieu Luria-Bertani et déposé $100 \mu \mathrm{L}$ du milieu de lavage sur des plaques de culture à la gélose Luria-Bertani. Nous avons compté les colonies après 24 heures d'incubation à $37^{\circ} \mathrm{C}$ (figure 1 ). Nous avons également ajouté $9,1 \mathrm{~mL}$ de milieu Luria-Bertani aux $900 \mu \mathrm{l}$ de milieu de lavage restants contenant les échantillons de $\mathrm{N} 95$, puis avons fait incuber le tout à $37^{\circ} \mathrm{C}$ dans un bain-marie à agitation pour cultures. Nous avons mesuré la densité optique à une longueur d'onde de $600 \mathrm{~nm}$ (spectrophotomètre microplaques Epoch, de BioTek) après 24 heures pour estimer la concentration bactérienne.

\section{Analyse microstructurelle du filtre des N95}

Afin de déterminer si l'exposition du filtre des N95 à une température élevée en dégradait les microfibres de polymère, nous avons analysé des échantillons de filtre de $1 \mathrm{~cm}^{2}$ venant de respirateurs non traités et de respirateurs ayant subi 10 désinfections thermiques à $70{ }^{\circ} \mathrm{C}$ et à une humidité relative de $0 \%$ ou de $50 \%$. Chaque échantillon a été enduit de $10 \mathrm{~nm}$ de carbone, puis examiné à l'aide d'un microscope électronique à balayage (XL30, de FEI) à un grossissement de 150 fois, de 200 fois, de 650 fois et de 1200 fois, à $5 \mathrm{keV}$. Une analyse morphologique de 10 fibres sélectionnées aléatoirement dans les divers quadrants d'une 

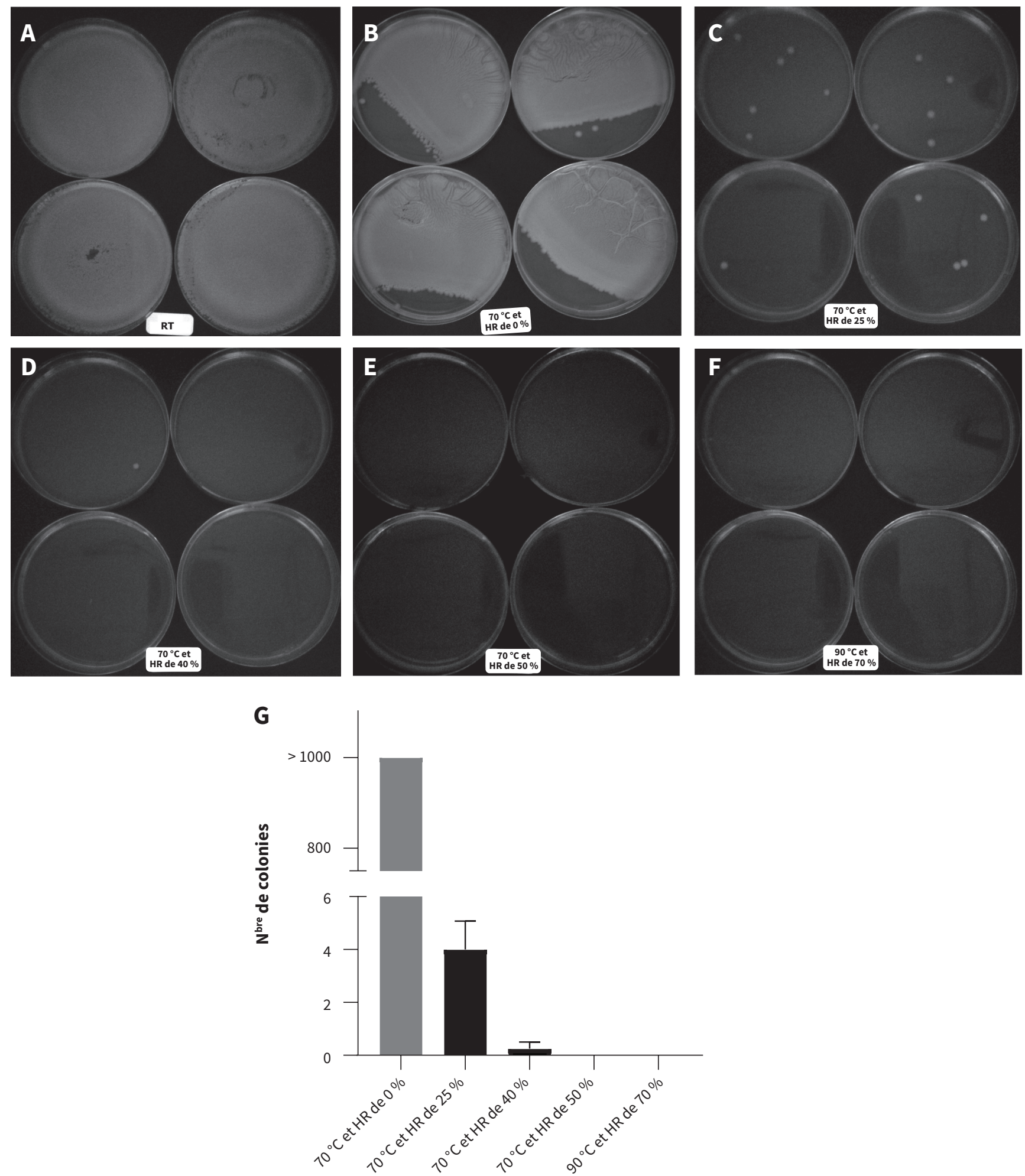

\section{Protocole de désinfection thermique}

Figure 1: Inactivation bactérienne sur les respirateurs N95 soumis à une désinfection thermique. A-F) Colonies d'Escherichia coli obtenues après l'incubation pendant 24 heures à $37^{\circ} \mathrm{C}$, d'échantillons issus de respirateurs contaminés ayant subi une désinfection thermique à une température et à une humidité relative variables. Pour chaque condition, nous avons utilisé 4 échantillons d'un même modèle de respirateur (1860S; de 3M) contaminé à $E$. coli $(n=20)$. Parmi tous les échantillons, 4 ont été laissés à température ambiante (témoins positifs, image A), et 20 ont subi une désinfection thermique dans des conditions variables, par groupes de $n=4$; B) $70{ }^{\circ} \mathrm{C}$ et humidité relative (HR) de $0 \%$; C) $70{ }^{\circ} \mathrm{C}$ et $\mathrm{HR}$ de $25 \%$; D) $70{ }^{\circ} \mathrm{C}$ et $\mathrm{HR}$ de $40 \%$; E) $70{ }^{\circ} \mathrm{C}$ et $\mathrm{HR}$ de $50 \%$; F) $90{ }^{\circ} \mathrm{C}$ et $\mathrm{HR}$ de $70 \%$, pour comparaison avec une température élevée. G) Numération des colonies d'E. coli issues de ces échantillons après 24 heures d'incubation à $37^{\circ} \mathrm{C}$. Les résultats montrent que 60 minutes de traitement à $70^{\circ} \mathrm{C}$ et à une $\mathrm{HR}$ de $50 \%$ inactivent efficacement $E$. coli sur les respirateurs contaminés. 
image représentative de chaque échantillon a été réalisée à l'insu avec ImageJ (https://imagej.nih.gov/ij/). Nous avons aussi mesuré le diamètre de 40 fibres par condition (10 fibres par type de masque) afin de calculer le diamètre moyen des fibres après chaque cycle de désinfection.

\section{Essai d'ajustement quantitatif des respirateurs N95}

L'exposition des respirateurs à des températures élevées pourrait avoir une incidence sur les propriétés mécaniques de leurs composants, par exemple sur l'élasticité des serre-tête et l'ajustabilité du pince-nez, ce qui risque de permettre le passage de particules. Pour évaluer l'étanchéité des respirateurs, nous avons fait un essai d'ajustement quantitatif normalisé pour 46 respirateurs ( $n=12$ par type de respirateur, sauf pour le $1860 \mathrm{~S}$, où $n=10$ ) au moyen du testeur d'ajustement Portacount Pro+ 8038 (TSI Incorporated) et du générateur de particules 8026 (TSI Incorporated) selon les directives réglementaires gouvernementales en vigueur (Health and Safety Executive du Royaume-Uni, Occupational Safety and Health Administration [OSHA] des États-Unis et Association canadienne de normalisation $[\mathrm{CSA}])^{11-13}$. La concentration des particules de plus de $0,02 \mu \mathrm{m}$ de diamètre détectées allait de 0,01 à 2,5 $\times 105$ particules $/ \mathrm{cm}^{3}$. Nous avons mesuré la concentration moyenne des particules dans l'air ambiant et sur les masques durant les essais normalisés, puis en avons calculé le ratio, qui correspond au facteur d'ajustement; selon l'OSHA, le seuil de passage est un facteur de $100^{11}$. Les respirateurs ont été ajustés pour 2 participants ( 1 homme et 1 femme) lors d'un test à l'insu, puis ont subi une désinfection thermique à une humidité relative de $0 \%$ ou de $50 \%$ ( $n=23$ pour chaque condition), comme susmentionné. Après 5,10 et 15 cycles de désinfection, nous avons refait un essai d'ajustement quantitatif pour chaque respirateur, avec les mêmes participants. Ceux-ci ont aussi noté l'ajustement subjectif, l'ajustabilité et le confort de chaque respirateur décontaminé, par rapport au respirateur original, selon l'échelle d'évaluation du confort de la CSA ( 0 = aucun inconfort, 1 = léger inconfort qui peut être ignoré, 2 = certain inconfort, mais qui ne nuit pas à l'exécution des tâches, $4=$ inconfort inacceptable $)^{13}$.

\section{Analyse de l'efficacité du filtre et de la résistance respiratoire des $\mathrm{N} 95$}

Nous nous sommes servis de la norme abrégée du National Institute for Occupational Safety and Health (NIOSH) pour évaluer la résistance respiratoire et l'efficacité de la filtration particulaire de $n=12$ masques non traités et de $n=58$ respirateurs N95 ayant subi 5 ou 10 cycles de désinfection thermique à une humidité relative de $0 \%$ ou de $50 \%{ }^{14-16}$. Pour mesurer la résistance respiratoire, nous avons fixé les respirateurs sur un appareil de test ayant un débit d'air de $85 \pm 2 \mathrm{~L} / \mathrm{min}$. Selon la norme du NIOSH, une résistance respiratoire inférieure à 343,23 Pa est jugée acceptable ${ }^{16}$. Pour évaluer l'efficacité de la filtration selon les protocoles du NIOSH, nous avons prétraité les respirateurs à une humidité relative de $85 \pm 5 \%$ et à une température de $38 \pm 2,5^{\circ} \mathrm{C}$ pendant $25 \pm 1$ heures, puis les avons fixés sur un compteur de particules à condensation homologué (modèle 3772, de TSI Incorporated). Nous avons testé les respirateurs à l'aide de billes de latex de polystyrène presque monodispersées à un débit de $85 \pm 2 \mathrm{~L} / \mathrm{min}$, à une température de 21 à $26^{\circ} \mathrm{C}$ et à une humidité relative de 30,4\% à 43,2 \%. L'efficacité de la filtration particulaire correspond au pourcentage des particules utilisées (diamètre médian : 0,075 $\pm 0,020 \mu \mathrm{m}$ ) qui sont retenues par le respirateur. Dans le cas des masques N95, elle doit être d'au moins $95 \%{ }^{15}$.

\section{Analyse statistique}

Nous avons effectué les analyses statistiques à l'aide du logiciel JMP (version 15.1.0, de SAS Institute) et calculé les statistiques descriptives de tous les respirateurs testés, pour chaque condition. Toutes les moyennes sont accompagnées d'un écart-type ( \pm ET). Comme la taille de l'échantillon était limitée en raison de la pénurie mondiale de respirateurs N95, nous avons effectué, pour chaque condition, un test $t$ pour échantillon unique afin de comparer les moyennes des groupes de masques désinfectés aux valeurs réglementaires américaines. Pour le critère d'ajustement quantitatif, nous avons calculé que la taille de l'échantillon devait être de $n=12$ pour détecter un facteur d'ajustement moyen de 120 (seuil de passage : 100; facteur d'ajustement moyen des masques non traités : $190 \pm 15$ ) avec un niveau a de 0,01 et une puissance de $0,95^{17}$. Nous avons opté pour un niveau a de 0,01 pour les valeurs $p$ unilatérales afin d'augmenter la rigueur de l'analyse et de tenir compte des comparaisons multiples. Par ailleurs, nous avons considéré que les masques désinfectés respectaient les normes réglementaires des États-Unis uniquement lorsque la limite inférieure de l'intervalle de confiance (IC) à $99 \%$ était supérieure au seuil de passage obligatoire. Voir le tableau 1 pour en savoir plus sur le modèle expérimental de l'étude et les méthodes statistiques employées.

\section{Résultats}

\section{Inactivation du SRAS-CoV-2}

Aucune particule infectieuse de SRAS-CoV-2 n'a pu être détectée sur les respirateurs préalablement contaminés puis traités à la chaleur sèche $\left(70{ }^{\circ} \mathrm{C}\right.$ pendant 60 minutes), alors que le virus était toujours en concentration élevée sur les respirateurs non traités (tableau 2).

\section{Inactivation bactérienne}

Aucune bactérie $E$. coli n'a été détectée sur les respirateurs N95 contaminés ayant subi un traitement à la chaleur de 60 minutes à une température de $70{ }^{\circ} \mathrm{C}$ et à une humidité relative de $50 \%$, ou à une température de $90{ }^{\circ} \mathrm{C}$ et à une humidité relative de $70 \%$. Comme le montre la figure 1 , plus de 1000 colonies bactériennes étaient toujours détectables dans les échantillons exposés à la chaleur sèche $\left(70{ }^{\circ} \mathrm{C}\right.$ et humidité relative de $\left.0 \%\right)$, tandis qu'elles étaient considérablement moins nombreuses dans ceux exposés à la chaleur humide $\left(70{ }^{\circ} \mathrm{C}\right.$ et humidité relative de $25 \%$ ou de $\left.40 \%\right)$. Bref, on peut dire que la désinfection thermique de 60 minutes à $70^{\circ} \mathrm{C}$ et à une humidité relative de $50 \%$ a éliminé $E$. coli des N95 (tableau 3, figure 1).

\section{Propriétés structurelles du filtre des N95}

Nous avons analysé le filtre de respirateurs N95 neufs et non traités (témoins) et avons constaté que le diamètre moyen des fibres était de 3,88 $\pm 2 \mu \mathrm{m}$. Même après 10 cycles de désinfection thermique de 60 minutes à $70^{\circ} \mathrm{C}$ et à une humidité relative 


\section{Tableau 1: Modèle expérimental*}

\begin{tabular}{|c|c|}
\hline Facteur évalué & $\begin{array}{l}\text { Modèle expérimental et taille de } \\
\text { l'échantillon }\end{array}$ \\
\hline $\begin{array}{l}\text { Inactivation du } \\
\text { SRAS-CoV-2 }\end{array}$ & $\begin{array}{l}3 \text { échantillons } \times 4 \text { modèles }= \\
12 \text { échantillons avec contamination virale } \\
\text { par condition (désinfection thermique de } \\
60 \text { minutes; désinfection thermique de } \\
60 \text { minutes avec refroidissement de } \\
5 \text { minutes à mi-cycle; aucune désinfection } \\
\text { thermique [témoins]) }\end{array}$ \\
\hline $\begin{array}{l}\text { Inactivation } \\
\text { bactérienne }\end{array}$ & $\begin{array}{l}4 \text { échantillons } \times 1 \text { modèle }(1860 \mathrm{~S} \text {, de } 3 \mathrm{M})= \\
4 \text { échantillons avec contamination } \\
\text { bactérienne par condition (air ambiant; } \\
70^{\circ} \mathrm{C} \text { et } \mathrm{HR} \text { de } 0 \% \text {, de } 25 \% \text {, de } 40 \% \text { et de } \\
50 \% \text {; } 90^{\circ} \mathrm{C} \text { et } \mathrm{HR} \text { de } 70 \% \text { ), et } \\
4 \text { échantillons } \times 1 \text { modèle ( } 1860 \mathrm{~S} \text {, de } 3 \mathrm{M} \text { ) = } \\
4 \text { échantillons témoins non contaminés à } \\
\text { l'air ambiant (témoins négatifs) }\end{array}$ \\
\hline $\begin{array}{l}\text { Analyse } \\
\text { microstructurelle } \\
\text { du filtre des N95 }\end{array}$ & $\begin{array}{l}10 \text { fibres pour } 1 \text { échantillon } \times 4 \text { modèles = } \\
40 \text { fibres pour chacune des } 3 \text { conditions } \\
\text { (non-traitement, } 10 \text { désinfections à une } \\
\text { HR de } 0 \% \text { et } 10 \text { désinfections à une HR de } \\
50 \% \text { ) }\end{array}$ \\
\hline
\end{tabular}

Ajustement quantitatif des respirateurs $\mathrm{N} 95$

Efficacité du filtre et résistance respiratoire

12 masques $\times 3$ modèles +10 masques $x$ 1 modèle $(1860 \mathrm{~S}$, de $3 \mathrm{M})=46$ respirateurs en tout; 23 d'entre eux ont subi des désinfections répétées $(5,10$ ou 15 cycles) à une $\mathrm{HR}$ de $0 \%$ ou de $50 \%$. Pour chaque condition, 2 participants ont testé 12 et 11 respirateurs, respectivement.
Critère d'évaluation

\section{Méthode statistique}

SRAS-CoV-2 $\left(\mathrm{DICT}_{50} / \mathrm{mL}\right)$ - Moyenne $\pm \mathrm{ET}$ pour chaque modèle et condition

Escherichia coli (densité optique à $600 \mathrm{~nm}$ et nombre de colonies)

Diamètre des fibres
Échelle d'évaluation du confort de la CSA, pour le facteur d'ajustement
Moyenne \pm ET pour chaque condition

- 2 tests $t$ bilatéraux indépendants pour comparer les moyennes des groupes et les témoins positifs
Efficacité de la filtration particulaire (pourcentage de particules retenues par le respirateur) et résistance respiratoire
3 masques $\times 4$ modèles $=12$ masques non traités; 3 masques $\times 4$ modèles $=$ 12 masques pour chaque condition traités à une $\mathrm{HR}$ de $50 \%$ ( 5 et 10 désinfections); 4 masques $\times 2$ modèles +5 masques $\times$ 2 modèles ( 8210 et $9105 \mathrm{~S}$, de $3 \mathrm{M})=$ 18 masques désinfectés 5 fois à une HR de $0 \%$; 4 masques $\times 4$ modèles $=16$ masques désinfectés 10 fois à une HR de $0 \%$
- Moyenne \pm ET du diamètre pour chaque condition (les modèles étaient munis de filtres électrets $3 \mathrm{M}$ semblables)

- Comparaison, au moyen d'un test $t$ unilatéral pour échantillon unique, des moyennes des groupes aux limites supérieure et inférieure de l'intervalle, selon le brevet américain, du diamètre des fibres des filtres de N95 non traités

- Facteur d'ajustement moyen \pm ET pour chaque condition

- Comparaison, pour chaque condition et au moyen d'un test $t$ unilatéral pour échantillon unique, des moyennes des groupes pour le facteur d'ajustement par rapport à la norme de 100 (seuil de passage défini par l'OSHA pour une protection respiratoire adéquate)

- Évaluation subjective de l'ajustement et du confort des respirateurs décontaminés, comparativement à des masques de référence neufs, selon l'échelle d'évaluation du confort de la CSA

- Moyennes de la filtration et de la résistance respiratoire $\pm E T$ pour chaque condition

- Comparaison, pour chaque condition et au moyen d'un test $t$ unilatéral pour échantillon unique, des moyennes des groupes pour l'efficacité de la filtration particulaire par rapport à la norme de $95 \%$ (seuil de passage pour les respirateurs N95 définie par le $\mathrm{NIOSH}$ )

- Comparaison, pour chaque condition et au moyen d'un test $t$ unilatéral pour échantillon unique, des moyennes des groupes pour la résistance respiratoire par rapport à la norme de 343,23 $\mathrm{Pa}$ (seuil de passage pour les respirateurs $\mathrm{N} 95$ définie par le NIOSH)

Remarque : $\mathrm{CSA}=$ Association canadienne de normalisation, $\mathrm{DICT}_{50} / \mathrm{mL}=$ dose infectieuse en culture tissulaire $50 \%$ par $\mathrm{mL}, \mathrm{ET}=$ écart-type, $\mathrm{HR}=$ humidité relative, $\mathrm{NIOSH}=\mathrm{National}$ Institute for Occupational Safety and Health, OSHA = Occupational Safety and Health Administration, SRAS-CoV-2 = coronavirus du syndrome respiratoire aigu sévère 2.

*Le tableau présente le modèle expérimental de l'étude, ce qui comprend le nombre d'échantillons pour chaque modèle de respirateur, le critère d'évaluation choisi et la méthode statistique utilisée. 
Titre, log $\mathrm{DICT}_{50} / \mathrm{mL}$ (moyenne $\left.\pm \mathrm{ET}\right)$

\begin{tabular}{|c|c|c|c|c|}
\hline $\begin{array}{l}\text { Modèle de N95 } \\
\text { (3M) }\end{array}$ & Prétraitement & $\begin{array}{l}\text { Témoins } \\
\text { (aucun traitement à la } \\
\text { chaleur) }\end{array}$ & $\begin{array}{c}70^{\circ} \mathrm{C} \text { et } \mathrm{HR} \text { de } 0 \% \text {, } \\
60 \mathrm{~min}\end{array}$ & $\begin{array}{l}70^{\circ} \mathrm{C} \text { et } \mathrm{HR} \text { de } 0 \%, \\
60 \mathrm{~min} \text { avec } \\
\text { refroidissement de } \\
5 \text { min à micycle }\end{array}$ \\
\hline \multirow[t]{2}{*}{$1860 \mathrm{~S}$} & Aucun & $5,62 \pm 0,21$ & Non détectable & Non détectable \\
\hline & $\begin{array}{l}10 \text { désinfections } \\
\text { thermiques }\end{array}$ & $5,69 \pm 0,11$ & Non détectable & Non détectable \\
\hline \multirow[t]{2}{*}{$8110 \mathrm{~S}$} & Aucun & $5,70 \pm 0,004$ & Non détectable & Non détectable \\
\hline & $\begin{array}{l}10 \text { désinfections } \\
\text { thermiques }\end{array}$ & $5,77 \pm 0,24$ & Non détectable & Non détectable \\
\hline \multirow[t]{2}{*}{$8210 \mathrm{~S}$} & Aucun & $5,21 \pm 0,50$ & Non détectable & Non détectable \\
\hline & $\begin{array}{l}10 \text { désinfections } \\
\text { thermiques }\end{array}$ & $5,66 \pm 0,08$ & Non détectable & Non détectable \\
\hline \multirow[t]{2}{*}{$9105 \mathrm{~S}$} & Aucun & $5,56 \pm 0,27$ & Non détectable & Non détectable \\
\hline & $\begin{array}{l}10 \text { désinfections } \\
\text { thermiques }\end{array}$ & $5,45 \pm 0,29$ & Non détectable & Non détectable \\
\hline \multicolumn{5}{|c|}{ 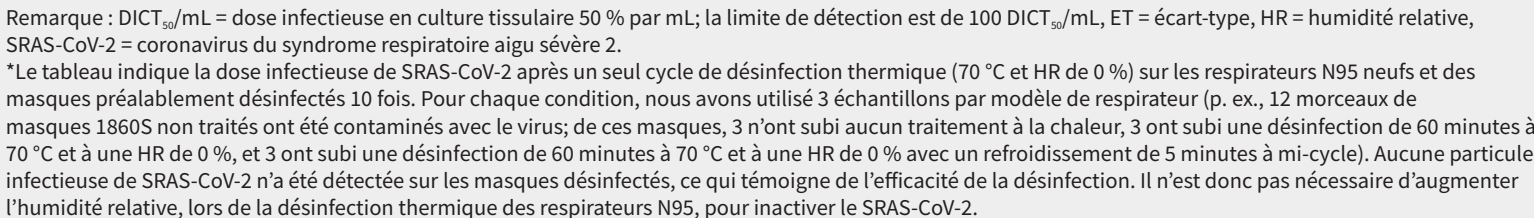 } \\
\hline
\end{tabular}

\section{Tableau 3: Quantification bactérienne sur les respirateurs N95 après une désinfection thermique, établie par} mesure de la densité optique à 600 nm après une culture de 24 heures*

\begin{tabular}{|c|c|c|c|c|c|c|c|}
\hline $\begin{array}{l}\text { No } \\
\text { d'échantillon }\end{array}$ & $\begin{array}{l}\text { Témoin } \\
\text { négatif }\end{array}$ & $\begin{array}{c}\text { Air } \\
\text { ambiant }\end{array}$ & $\begin{array}{c}70{ }^{\circ} \mathrm{C} \text { et HR } \\
\text { de } 0 \%\end{array}$ & $\begin{array}{c}70{ }^{\circ} \mathrm{C} \text { et HR } \\
\text { de } 25 \%\end{array}$ & $\begin{array}{c}70{ }^{\circ} \mathrm{C} \text { et } \mathrm{HR} \\
\text { de } 40 \%\end{array}$ & $\begin{array}{c}70{ }^{\circ} \mathrm{C} \text { et HR } \\
\text { de } 50 \%\end{array}$ & $\begin{array}{c}90{ }^{\circ} \mathrm{C} \text { et HR } \\
\text { de } 70 \%\end{array}$ \\
\hline 1 & 0 & 2,777 & 2,875 & 2,74 & 2,84 & 0 & 0 \\
\hline 2 & 0,001 & 2,851 & 2,861 & 2,625 & 2,768 & 0,04 & 0 \\
\hline 3 & 0,002 & 2,816 & 2,912 & 2,709 & 2,733 & 0,02 & 0 \\
\hline 4 & 0 & 2,64 & 2,736 & 2,748 & 0 & 0 & 0 \\
\hline Moyenne $\pm \mathrm{ET}$ & $0,00 \pm 0,00$ & $2,77 \pm 0,09$ & $2,84 \pm 0,08$ & $2,71 \pm 0,06$ & $2,09 \pm 1,39$ & $0,02 \pm 0,02$ & $0,00 \pm 0,00$ \\
\hline Valeur $p \nmid$ & $<0,001$ & - & 0,842 & 0,863 & 0,081 & $<0,001$ & $<0,001$ \\
\hline \multicolumn{8}{|c|}{$\begin{array}{l}\text { Remarque : } E \text {. coli }=\text { Escherichia coli, ET = écart type, HR = humidité relative. } \\
\text { *Le tableau présente les résultats de la quantification d'E. coli, établis par mesure de la densité optique à } 600 \text { nm. Pour chaque condition, nous avons utilisé } \\
4 \text { échantillons d'un modèle ( }(1860 \mathrm{~S} \text {, de } 3 \mathrm{M}) \text {. Les témoins négatifs ont été « contaminés » avec un milieu Luria-Bertani pur, puis laissés à l'air ambiant. Parmi les } \\
\text { échantillons de respirateurs contaminés à } E \text {. coli ( } n=24), 4 \text { ont été laissés à l'air ambiant (témoins positifs), et } 20 \text { ont subi une désinfection thermique dans des } \\
\text { conditions variables }\left(70^{\circ} \mathrm{C} \text { à une } \mathrm{HR} \text { de } 0 \% \text {, de } 25 \% \text {, de } 40 \% \text { ou de } 50 \% \text {, et } 90^{\circ} \mathrm{C} \text { à une } \mathrm{HR} \text { de } 70 \% ; n=4 \text { par condition). Les données montrent que la désinfection }\right. \\
\text { thermique élimine } E \text {. coli lorsque l'humidité relative est d'au moins } 50 \% \text {, mais pas lorsqu'elle est moindre. } \\
\text { †Comparativement aux témoins positifs (échantillons contaminés à } E \text {. coli laissés à l'air ambiant). }\end{array}$} \\
\hline
\end{tabular}

de $0 \%$ ou de $50 \%$, le diamètre moyen des fibres demeurait dans les limites des filtres de N95 non traités précisées dans le brevet américain (figure 2$)^{9}$.

\section{Autres propriétés des respirateurs}

Nous avons effectué des essais d'ajustement quantitatifs sur 4 types de respirateurs N95 couramment offerts sur le marché après 5,10 et 15 cycles de désinfection thermique à une humidité relative de $0 \%$ ou de $50 \%$ ( $n=23$ pour chaque condition; figure 3 ). Tous les groupes de respirateurs soumis à une désinfection thermique dépassaient de beaucoup le facteur d'ajustement de 100 , soit la norme de passage de l'OSHA indiquant une protection respiratoire adéquate ( $p<0,001$ pour tous les groupes), ainsi que la limite inférieure des IC à 99 \% (figure 3). Aucun des respirateurs 

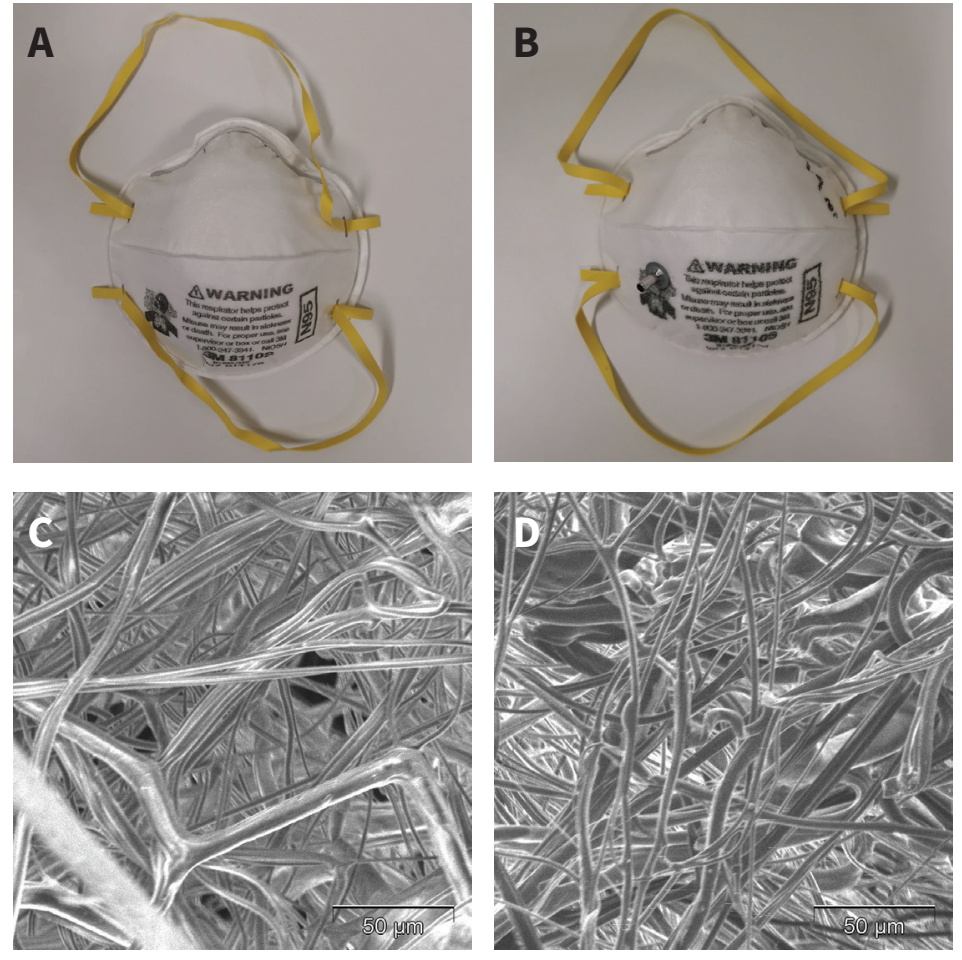

$\mathbf{E}$

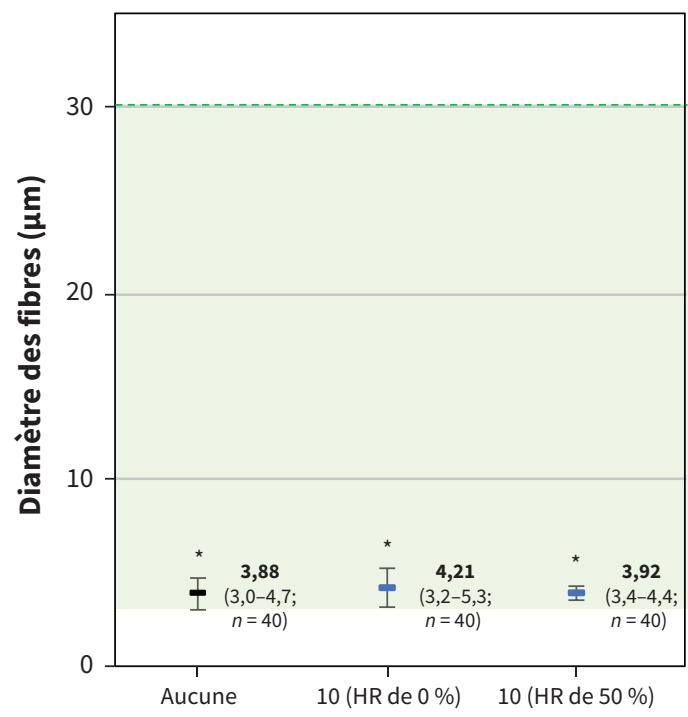

$\mathrm{N}^{\text {bre }}$ de désinfections

Figure 2: Effet de la désinfection thermique sur les propriétés structurelles des respirateurs N95. A et C) Respirateur N95 non traité; B et D) Respirateur N95 ayant subi 10 désinfections thermiques à une humidité relative (HR) de $50 \%$ (dans les 2 cas : modèle $8110 \mathrm{~S}$ de $3 \mathrm{M}$ ). C et D) Filtre de N95 grossi 650 fois (microscopie électronique à balayage; échelle : $50 \mu \mathrm{m}$ ). E) Diamètre des fibres des filtres de N95 non traités et de N95 désinfectés 10 fois (HR de $0 \%$ et de $50 \%$ ), et intervalle - selon le brevet américain - du diamètre des fibres de filtres de N95 neufs (non traités) de 3M ombragé en vert ${ }^{9}$. Les moyennes des groupes de masques non traités et de masques désinfectés 10 fois sont significativement plus basses que la limite supérieure de cet intervalle ( $p<0,001$ pour tous les groupes) et significativement plus élevées que la limite inférieure (masques non traités: $p=0,004$; masques désinfectés 10 fois à une HR de $0 \%: p=0,002$; et masques désinfectés 10 fois à une HR de $50 \%: p<0,001$ ). Les groupes qui diffèrent significativement des 2 limites sont marqués d'un astérisque ( $\left.{ }^{*} p<0,01\right)$. Nous avons retenu 1 échantillon pour chacun des 4 modèles de N95 testés, puis avons mesuré 10 fibres choisies aléatoirement pour chaque échantillon (40 fibres par condition). Le diamètre moyen des fibres, avec un intervalle de confiance (IC) à $99 \%$, est présenté sous forme de barres d'erreur. La moyenne est également inscrite, avec l'IC à $99 \%$ et le nombre de fibres mesurées entre parenthèses.

désinfectés n'a échoué le test, sur les 138 essais d'ajustement quantitatifs réalisés. Par ailleurs, l'ajustement subjectif et le confort des respirateurs décontaminés demeuraient comparables à ceux des masques neufs, et ont été évalués à 0 (aucun inconfort) sur l'échelle d'évaluation du confort de la CSA.

De plus, nous avons testé l'efficacité de la filtration particulaire et la résistance respiratoire de respirateurs N95 - les 4 mêmes types - ayant subi 5 ou 10 cycles de désinfection thermique à une humidité relative de $0 \%$ ou de $50 \%$ (figure 3). L'efficacité de filtration des respirateurs désinfectés était considérablement supérieure à $95 \%$ après 5 et 10 cycles de désinfection $(p<0,001)$. En outre, pour tous les groupes de respirateurs testés, la résistance respiratoire était nettement inférieure à la norme maximale acceptable de 343,23 $\mathrm{Pa}(p<0,001)$.

\section{Interprétation}

Étant donné les récentes études mettant en évidence la sensibilité à la chaleur du SRAS-CoV-2, nous avons décidé de soumettre 4 modèles courants de respirateurs N95 à usage unique à une désinfection thermique ${ }^{5,8}$. Nous avons constaté qu'un seul cycle de désinfection thermique de 60 minutes à $70^{\circ} \mathrm{C}$ et à une humidité relative de $0 \%$ inactivait efficacement le SRAS-CoV-2 sur les 4 types de masques, et que le réglage de l'humidité relative à $50 \%$ ou plus éliminait également $E$. coli. Nous avons aussi observé que la désinfection thermique n'altérait pas la structure du filtre électret des respirateurs et qu'elle n'influait pas sur l'ajustement, la filtration, la résistance respiratoire et le confort après au moins 10 cycles à une humidité relative de $0 \%$ ou de $50 \%$.

Comme il y a une grande demande d'EPI durant la pandémie de COVID-19, les travailleurs de première ligne sont souvent appelés à réutiliser des N95 à usage unique ${ }^{4,18}$. Des stratégies visant la désinfection et la redistribution de N95 assignés augmenteraient la sécurité des travailleurs de la santé et ralentiraient l'épuisement des stocks. Or, jusqu'à présent, il n'existait aucun protocole de décontamination à grande échelle des N95 qui soit sûr et universellement accessible.

Pour réagir efficacement à la pénurie mondiale d'EPI, il faut largement diffuser des stratégies de désinfection et de réutilisation. La Food and Drug Administration des États-Unis a récemment autorisé la stérilisation en urgence de respirateurs N95 à usage unique au moyen de peroxyde d'hydrogène vaporisé21,22. Cette substance inactive le SRAS-CoV-2 et d'autres agents pathogènes sur les respirateurs N95 tout en préservant l'ajustement quantitatif de ces derniers pour au moins 3 cycles de décontamination ${ }^{23,24}$. Cette 
A Essai d'ajustement quantitatif
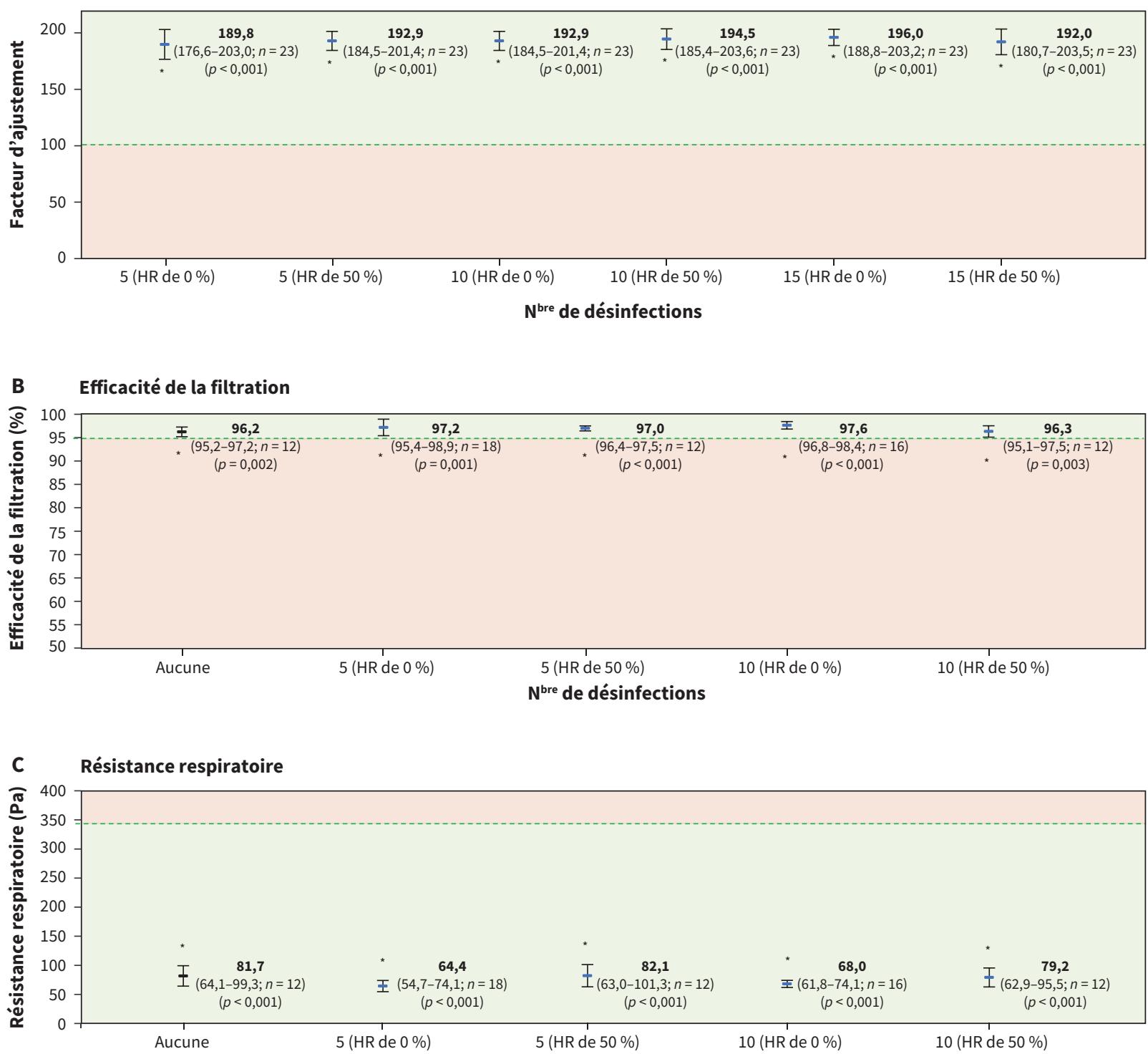

$N^{\text {bre }}$ de désinfections

Figure 3: Propriétés des respirateurs N95 ayant subi une désinfection thermique. A) Facteurs d'ajustement quantitatif des respirateurs N95 soumis à 5 , 10 ou 15 cycles de désinfection thermique à une humidité relative (HR) de $0 \%$ ou de $50 \%$ ( $n=23$ masques par condition [5 masques du modèle $1860 \mathrm{~S}$ et 6 masques de chacun des 3 autres modèles]). Le facteur d'ajustement de 100, défini par l'Occupational Safety and Health Administration comme étant le seuil de passage, est représenté par une ligne traitillée ${ }^{11}$. B) Efficacité de la filtration particulaire (pourcentage de particules retenues par le respirateur). C) Résistance respiratoire (résistance au flux d'air) des respirateurs N95 non traités ( $n=12$ masques par condition [3 masques par modèle]) et des respirateurs N95 soumis à 5 ou 10 cycles de désinfection thermique à une HR de $0 \%$ ou de $50 \%$ (masques désinfectés 5 fois à une HR de $0 \%: n=18$ [ 4 masques pour chacun des modèles $8110 \mathrm{~S}$ et $1860 \mathrm{~S}$ de 3M, et 5 masques pour chacun des modèles 8210 et $9105 \mathrm{~S}$, aussi de 3M]; masques désinfectés 10 fois à une HR de $0 \%: n=16$ [4 masques par modèle]; masques désinfectés 5 et 10 fois à une HR de $50 \%: n=12$ [ 3 masques par modèle]). Les seuils de passages fixés par le National Institute for Occupational Safety and Health (efficacité de filtration $\geq 95 \%$ et résistance respiratoire $\leq 343,23$ Pa) sont représentées par une ligne traitillée ${ }^{15,16}$. Les données sont présentées sous forme de moyennes, avec l'intervalle de confiance (IC) à $99 \%$ et la taille de l'échantillon indiqués entre parenthèses. Les groupes qui surpassent de façon significative le seuil de passage sont marqués d'un astérisque $\left({ }^{\star} p<0,01\right)$. La valeur $p$ comparant la moyenne du groupe et la valeur réglementaire américaine officielle est indiquée pour chaque paramètre.

technologie est toutefois limitée aux respirateurs qui ne sont pas à base de cellulose, ce qui élimine une bonne partie des N95, et est inaccessible dans la plupart des hôpitaux et des autres établissements ${ }^{25}$. La désinfection thermique, quant à elle, peut être réalisée à faible coût à l'aide de fours à convection mécanique classiques, que l'on trouve couramment dans les cuisines commerciales, les laboratoires et les installations de stérilisation. La grande capacité de ces fours permet la désinfection de milliers de masques par appareil par jour, ce qui pourrait favoriser l'adoption de la technique à une échelle suffisante 
pour accroître les stocks mondiaux d'EPI. La désinfection thermique pourrait donc être une solution envisageable dans certaines régions à revenu faible ou intermédiaire, où l'accès à l'EPI et les capacités de dépistage sont limités, pour protéger les travailleurs de première ligne pendant la pandémie.

Une étude récente est venue appuyer nos conclusions en montrant l'inactivation du SRAS-CoV-2 sur des respirateurs N95 exposés pendant 60 minutes à une chaleur sèche de $70{ }^{\circ} \mathrm{C}^{23}$. Selon d'autres études, les désinfections thermiques plus courtes (15 à 40 min à une température de $75^{\circ} \mathrm{C}$ à $100{ }^{\circ} \mathrm{C}$ et à une humidité relative de $0 \%$ à $100 \%$ ) préservent l'ajustement et la capacité de filtration des $\mathrm{N} 95$ pendant de nombreux cycles, mais leur efficacité antivirale n'a pas été testée ${ }^{26-29}$.

Outre la désinfection thermique, d'autres procédures de décontamination ont été étudiées, dont le rayonnement ultraviolet (UV) [250 à $280 \mathrm{~nm}$ ], la stérilisation en autoclave et des traitements chimiques à base d'éthanol à $70 \%$ ou de solutions chlorées à $2 \%$ (quelques références sont des prépublications non révisées par des pairs) $)^{23,28-35}$. Il a été démontré que la stérilisation en autoclave et les traitements chimiques réduisent rapidement la capacité de filtration des respirateurs N95 et, par conséquent, ne sont pas appropriés pour une utilisation clinique $\mathrm{e}^{23,28,34-36}$. En revanche, la décontamination par rayonnement UV pourrait être une approche prometteuse puisqu'elle semble préserver les propriétés des respirateurs et inactiver le SRAS-CoV-2 ${ }^{23,33}$. Son adaptabilité pourrait toutefois être limitée, l'empilage des masques et les ombres qu'il crée pouvant nuire à la pénétration des rayons UV dans le matériau poreux qui constitue le filtre des $\mathrm{N}^{28}{ }^{28}$.

Utilisée de pair avec d'autres méthodes de traitement, la désinfection thermique peut servir de mesure d'urgence pour pallier rapidement la pénurie mondiale de respirateurs N95. Des études ultérieures pourraient comparer la sûreté, l'adaptabilité et la rentabilité des diverses méthodes de décontamination des N95 et, pour mieux évaluer la sûreté des méthodes en contexte réel, se pencher sur l'inactivation du SRAS-CoV-2 dans les respirateurs contaminés par des liquides biologiques comme la salive ou le sang.

\section{Limites de l'étude}

Étant donné la pénurie mondiale de respirateurs N95, la taille de l'échantillon étudié était limitée. La réalisation d'une étude de noninfériorité classique comparant des masques désinfectés à des masques non traités aurait nécessité un échantillon plus important (p. ex., 155 masques par groupe pour avoir une limite de non-infériorité de 5 pour le facteur d'ajustement, à une puissance de 0,9 et à une valeur a de 0,05) ou des marges de non-infériorité larges et exagérées. Nous avons donc déterminé la sûreté des masques désinfectés en comparant les caractéristiques aux critères d'approbation des N95 de l'OSHA et du NIOSH. À noter que lors de la prestation de soins aux patients atteints de la COVID-19, il est possible que les N95 soient contaminés par des liquides biologiques infectés, comme du sang, et qu'une exposition prolongée à la chaleur soit nécessaire pour inactiver le virus. C'est pourquoi nous avons porté le temps d'exposition à 60 minutes et que nous recommandons de ne pas traiter les masques visiblement contaminés. Autre limite potentielle de l'étude : nous n'avons pas examiné séparément tous les composants des respirateurs (bandes élastiques, etc.) pour y vérifier l'inactivation totale du virus. Cependant, les virus se trouvant dans le filtre électret, qui a été testé, sont plus susceptibles de résister à la désinfection thermique que ceux se trouvant sur les autres composants du masque; il est donc peu probable que les bandes élastiques et les autres composants portent une charge infectieuse après une désinfection thermique.

\section{Conclusion}

La désinfection thermique pendant 60 minutes à $70{ }^{\circ} \mathrm{C}$ inactive le SRAS-CoV-2. La méthode utilisée ne demande que de l'équipement largement accessible et permet la réutilisation sûre des respirateurs N95 à usage unique, sans compromettre leur effet protecteur. Compte tenu de l'inactivation complète du SRAS-CoV-2 et de l'inactivation bactérienne supérieure observées, nous proposons d'opter pour la désinfection thermique à une humidité relative de $50 \%$, jusqu'à un maximum de 10 cycles. Cette intervention pourrait être réalisable, efficace et rapidement adaptable dans les régions peu technologiques, où elle aiderait à protéger les travailleurs de première ligne contre l'infection dans leur milieu de travail durant la pandémie.

\section{Références}

1. Coronavirus disease 2019 (COVID-19) situation report - 82. Genève : Organisation mondiale de la Santé; 2020. Accessible ici : www.who.int/docs/default-source/ coronaviruse/situation-reports/20200411-sitrep-82-covid-19.pdf?sfvrsn=74a5d15_2 (consulté le 11 avril 2020).

2. COVID-19 Taskforce of the Department of Infectious Diseases and the IT Service. Integrated surveillance of COVID-19 in Italy. Rome (Italie) : L'epidemiologia per la sanità pubblica, Istituto Superiore di Sanità; 2020. Accessible ici : www.epicentro. iss.it/en/coronavirus/bollettino/Infografica_10aprile\%20ENG.pdf (consulté le 11 avril 2020).

3. Utilisation rationnelle des équipements de protection individuelle (EPI) contre la maladie à coronavirus 2019 (COVID-19) et éléments à considérer en cas de grave pénurie. Genève : Organisation mondiale de la Santé; 2020. Accessible ici : https://apps.who.int/iris/handle/10665/331765 (consulté le 11 avril 2020).

4. Recommended guidance for extended use and limited reuse of N95 filtering facepiece respirators in healthcare settings. Cincinnati : National Institute for Occupational Safety and Health (NIOSH), Centers for Disease Control and Prevention; 2020. Accessible ici : https://www.cdc.gov/niosh/topics/hcwcontrols/ recommendedguidanceextuse.html (consulté le 11 avril 2020).

5. Chin AWH, Chu JTS, Perera MRA, et al. Stability of SARS-CoV-2 in different environmental conditions. Lancet Microbe 2020;1:e10. doi : 10.1016/S2666-5247(20)30003-3.

6. Casanova L, Rutala WA, Weber DJ, et al. Coronavirus survival on healthcare personal protective equipment. Infect Control Hosp Epidemiol 2010;31:560-1.

7. Decontamination of $3 \mathrm{M}$ filtering facepiece respirators, such as N95 respirators, in the United States: considerations. 8e révision [bulletin technique]. Saint Paul (Minnesota) : 3M Company; 2020. Accessible ici : https://multimedia.3m. $\mathrm{com} / \mathrm{mws} /$ media/18248690/decontamination-methods-for-3m-n95-respirators -technical-bulletin.pdf (consulté le 6 mai 2020).

8. Domingo MC. Inactivation thermique du virus SARS-CoV-2 dans des échantillons cliniques. Quebec Indlsp. IDBM : Institut national de la santé publique du Québec; 2020. Accessible ici : www.inspq.qc.ca/sites/default/files/lspq/rapport -inactivation_thermique_sars-cov-2.pdf (consulté le 11 avril 2020).

9. Angadjivand SA, Jones ME et Meyer DE, inventeurs; 3M Company, cessionnaire. Electret filter media. États-Unis [brevet]; 2000. Accessible ici : https://patents. justia.com/patent/6119691 (consulté le 11 avril 2020).

10. Windholz M, dir. The Merck index: an encyclopedia of chemicals and drugs. 9e édition. Rahway (New Jersey) : Merck \& Co. Inc.; 1976.

11. Appendix A to $§ 1910.134$ - Fit testing procedures (mandatory). Part 1. OSHAaccepted fit test protocols. A. Fit testing procedures - general requirements. Washington (District de Columbia) : Occupational Safety and Health Administration; 2020. Accessible ici : www.osha.gov/laws-regs/regulations/standardnumber/ 1910/1910.134AppA (consulté le 11 avril 2020).

12. Guidance on respiratory protective equipment (RPE) fit testing. Dépliant INDG479. Health and Safety Executive; 2019. Accessible ici : www.hse.gov.uk/pubns/ indg479.pdf (consulté le 11 avril 2020). 
13. CSA Z94.4:F18 : Choix, utilisation et entretien des appareils de protection respiratoire. CSA Group; 2018:1-163. Accessible ici : https://store.csagroup.org/ ccrz_ProductDetails?viewState=DetailView\&cartID=\&sku=CAN/CSA-Z94.4-18 \&isCSRFlow=true\&portalUser=\&store=\&cclcl=fr_CA (consulté le 11 avril 2020).

14. National Institute for Occupational Safety and Health, National Personal Protective Technology Laboratory. Determination of particulate filter efficiency level for N95 series filters against solid particulates for non-powered, air-purifying respirators standard testing procedure (STP). Procédure no TEB-APR-STP-0059. Révision : 3.2. Pittsburgh (Pennsylvanie) : National Personal Protective Technology Laboratory (NPPTL), Centers for Disease Control and Prevention; 2019. Accessible ici : www.cdc.gov/niosh/npptl/stps/pdfs/TEB-APR-STP-0059-508.pdf (consulté le 11 avril 2020).

15. 42 CFR 84.181: Non-powered air-purifying particulate filter efficiency level determination. Washington (District de Columbia) : Government Publishing Office des États-Unis; 2009.

16. 42 CFR 84.180: Airflow resistance tests. Washington (District de Columbia) : Government Publishing Office des États-Unis; 2009

17. Faul F, Erdfelder E, Lang A-G, et al. G*Power 3: a flexible statistical power analysis program for the social, behavioral, and biomedical sciences. Behav Res Methods 2007;39:175-91.

18. Summary for healthcare facilities: strategies for optimizing the supply of N95 respirators during the COVID-19 response. Atlanta : Centers for Disease Control and Prevention; Mise à jour le 16 avril 2020. Accessible ici : www.cdc.gov/ coronavirus/2019-ncov/hcp/checklist-n95-strategy.html (consulté le 10 avril 2020).

19. Huang S-H, Chen C-W, Kuo Y-M, et al. Factors affecting filter penetration and quality factor of particulate respirators. Aerosol Air Qual Res 2013;13:162-71. doi : 10.4209/aaqr.2012.07.0179.

20. Lin $\mathrm{T}-\mathrm{H}, \mathrm{Chen} \mathrm{C}-\mathrm{C}$, Huang $\mathrm{S}-\mathrm{H}$, et al. Filter quality of electret masks in filtering 14.6-594 $\mathrm{nm}$ aerosol particles: effects of five decontamination methods. PLOS One 2017;12:e0186217.

21. Hinton DM. Emergency Use Authorization (EUA) for the emergency use of the Advanced Sterilization Products, Inc. (ASP) STERRAD 100S, NX, and 100NX Sterilization Systems. Rockville (Maryland) : Food and Drug Administration des États-Unis; 6 juin 2020. Accessible ici : www.fda.gov/media/136884/download (consulté le 15 avril 2020).

22. Emergency Use Authorization (EUA) for the emergency use of Stryker Instrument's Sterizone VP4 Sterilizer. Rockville (Maryland) : Food and Drug Administration des États-Unis; 6 juin 2020. Accessible ici : www.fda.gov/media/136976/download (consulté le 16 avril 2020).

23. Fischer RJ, Morris DH, van Doremalen N, et al. Effectiveness of N95 respirator decontamination and reuse against SARS-CoV-2 virus. Emerg Infect Dis 3 juin 2020;26 [Cyberpublication avant impression]. doi : 10.3201/ eid2609.201524.
24. Ibáñez-Cervantes G, Bravata-Alcántara JC, Nájera-Cortés AS, et al. Disinfection of N95 masks artificially contaminated with SARS-CoV-2 and ESKAPE bacteria using hydrogen peroxide plasma: impact on the reutilization of disposable devices. Am J Infect Control 6 juillet 2020 [Cyberpublication avant impression]. S0196-6553(20)30639-8. doi : 10.1016/j.ajic.2020.06.216.

25. Fact sheet for healthcare personnel on emergency use of STERRAD ${ }^{\circledR}$ sterilization systems to reprocess N95 respirators. Irvine (Californie) : Advanced Sterilization Products; 2020. Accessible ici : www.asp.com/sites/default/files/covid-19 /2000014-Fact_Sheet_for_Healthcare_Personnel_on_Emergency_Use_of_STERRAD Sterilization_Systems_to_Reprocess_N95_Respirators.pdf (consulté le 6 mai 2020).

26. Anderegg L, Meisenhelder C, Ngooi CO, et al. A scalable method of applying heat and humidity for decontamination of $\mathrm{N} 95$ respirators during the COVID-19 crisis. PLoS One 2020;15:e0234851.

27. Li DF, Cadnum JL, Redmond SN, et al. Steam treatment for rapid decontamination of N95 respirators and medical face masks. Am J Infect Control 2020;48:855-7.

28. Liao L, Xiao W, Zhao M, et al. Can $\mathrm{N} 95$ respirators be reused after disinfection? How many times? ACS Nano 2020;14:6348-56

29. Zulauf KE, Green AB, Nguyen Ba AN, et al. Microwave-generated steam decontamination of $\mathrm{N} 95$ respirators utilizing universally accessible materials. medRxiv 25 avril 2020. doi : 10.1101/2020.04.22.20076117.

30. Liao L, Xiao W, Zhao M, et al. Can N95 respirators be reused after disinfection? How many times? ACS Nano 2020;14:6348-56.

31. Kumar A, Kasloff SB, Leung A, et al. N95 mask decontamination using standard hospital sterilization technologies. medRiv 20 avril 2020 [Prépublication]. doi : 10.1101/2020.04.05.20049346.

32. Smith JS, Hanseler $\mathrm{H}$, Welle J, et al. Effect of various decontamination procedures on disposable N95 mask integrity and SARS-CoV-2 infectivity. medRiv 7 mai 2020 [Prépublication]. doi : 10.1101/2020.04.11.20062331.

33. Decontamination of $3 \mathrm{M}$ filtering facepiece respirators, such as N95 respirators, in the United States: considerations. 9e révision [bulletin technique]. Saint Paul (Minnesota) : 3M Company; 2020. Accessible ici : https://multimedia.3m.com/ mws/media/18248690/decontamination-methods-for-3m-filtering-facepiece -respirators-technical-bulletin.pdf (consulté le 11 juin 2020).

34. Grinshpun SA, Yermakov M, Khodoun M. Autoclave sterilization and ethanol treatment of re-used surgical masks and N95 respirators during COVID-19: impact on their performance and integrity. J Hosp Infect 2020;105:608-14.

35. Lin $\mathrm{T}-\mathrm{H}, \mathrm{Chen} \mathrm{C}-\mathrm{C}$, Huang $\mathrm{S}-\mathrm{H}$, et al. Filter quality of electret masks in filtering 14.6-594 $\mathrm{nm}$ aerosol particles: effects of five decontamination methods. PLoS One 2017;12:e0186217.

36. Grossman J, Pierce A, Mody J, et al. Institution of a novel process for N95 respirator disinfection with vaporized hydrogen peroxide in the setting of the COVID-19 pandemic at a large academic medical center. J Am Coll Surg 27 avril 2020 [Cyberpublication avant impression]. S1072-7515(20)30349-5. doi : 10.1016/j.jamcollsurg.2020.04.029.
Intérêts concurrents : Aucun intérêt concurrent n’a été déclaré.

Cet article a été révisé par des pairs.

Affiliations : Institut de recherche de l'Hôpital pour enfants malades (SickKids) (Daeschler, Chan, Tajdaran, Mirmoeini, Darbandi), Hôpital SickKids; Service de santé et sécurité au travail de l'Hôpital SickKids (Manson); Division de chirurgie plastique et reconstructive (Joachim, J. Zhang), Hôpital SickKids et Université de Toronto, Toronto (Ontario); École de santé publique, Faculté de médecine LKS (Chin, Poon), Université de Hong Kong, Pokfulam, Région administrative spéciale de Hong Kong, Chine; Département de génie chimique et de chimie appliquée (Chen, Gu), Université de Toronto; Département d'anesthésie et de médecine de la douleur (Maynes, L. Zhang), Division d'infectiologie (Science), Programme des sciences évaluatives de la santé des enfants (Stephens), Institut des biomatériaux et du génie biomédical et Division de chirurgie plastique et reconstructive (Borschel), Hôpital SickKids, Toronto (Ontario).

Collaborateurs : Simeon Daeschler, Niclas Manson, Gregory Borschel, Jason Maynes et Michelle Science ont contribué à l'élaboration et à la conception de l'étude. Simeon Daeschler, Niclas Manson, Paul Chen, Kiana Tajdaran, Kaveh Mirmoeini, Jennifer Zhang, Katelyn Chan, Ali Darbandi, Libo Zhang, Leo Poon et Alex Chin ont collaboré à l'acquisition des données. Simeon Daeschler et Derek Stephens ont réalisé l'analyse statistique. Simeon Daeschler, Niclas Manson et Gregory Borschel ont interprété les données et rédigé la première version du manuscrit. Tous les auteurs ont révisé de façon critique le contenu intellectuel important du manuscrit; ils ont donné leur approbation finale pour la version destinée à être publiée et assument l'entière responsabilité de tous les aspects du travail. Simeon Daeschler et Niclas Manson ont contribué à cet article à parts égales.

Financement : Des fonds ont été reçus du Health and Medical Research Fund (COVID190116) de Hong Kong, ainsi que du National Institute of Allergy and Infectious Diseases des National Institutes of Health (contrat HHSN272201400006C). Les bailleurs de fonds n'ont joué aucun rôle dans la conception de l'étude; la collecte, l'analyse et l'interprétation des données; la rédaction du rapport; et la prise de décisions relatives à la publication de l'article.

Partage des données : Tous les ensembles de données analysés peuvent être obtenus de l'auteur-ressource (gregory.borschel@sickkids.ca) si la demande est jugée raisonnable.

Remerciements : Les auteurs remercient Dianna McCluskey, Ilda Ponte, Lucia Couto et Louis Konstant pour l'assistance technique fournie lors du traitement des respirateurs N95.

Accepté : 15 juillet 2020

Correspondance : Gregory Borschel, gregory.borschel@sickkids.ca 